\title{
On Emerging Contractual Relationships for Local 5G Micro Operator Networks
}

\author{
Bidushi Barua \\ Centre for Wireless Communications \\ University of Oulu \\ Oulu, Finland \\ bidushi.barua@oulu.fi
}

\author{
Marja Matinmikko-Blue \\ Centre for Wireless Communications \\ University of Oulu \\ Oulu, Finland \\ marja.matinmikko@oulu.fi
}

\author{
Matti Latva-aho \\ Centre for Wireless Communications \\ University of Oulu \\ Oulu, Finland \\ matti.latva-aho@oulu.fi
}

\begin{abstract}
Local 5G micro operator (uO) networks are emerging to satisfy local capacity, coverage, and context specific service needs in certain geographical locations to complement Mobile Network Operators' (MNOs') offerings. Conventionally, MNOs have been reluctant to allow entry to local 5G micro operators (uOs) as the latter can turn out to be a threat for MNOs. For successful emergence of uOs into the future mobile market, it is necessary to define the features of contractual relationships that will arise between the existing MNOs, uOs and the users for different $\mathrm{uO}$ deployment scenarios. In this paper, we propose these contract features, different kinds of competition that will emerge, and pricing mechanisms for these deployments. Finally, a mathematical model is used to analyze the impact of competition among uOs on the equilibrium wholesale price where the wholesale price refers to the price that the MNO needs to the pay a $\mathrm{uO}$ for serving its customers. From our results, it is found that competition in these networks can put downward pressure on the wholesale price and therefore brings about a reduction in it.
\end{abstract}

Index Terms-Competition, contracts, micro operator networks, pricing.

\section{INTRODUCTION}

The current mobile communication market is dominated by cellular mobile network operators (MNOs) that deploy wide area networks. Upcoming $5 \mathrm{G}$ networks on the other hand, are increasingly targeting geographically constrained areas due to the location specific usage demand characteristics and operations in higher carrier frequencies [1]. These local deployments aim to serve areas with versatile services, in specific locations such as shopping malls, hospitals, stadiums, industry plants, etc. [2], where the requirements of mobile traffic are constantly increasing. For such local mobile traffic, the concept of micro operator $(\mathrm{uO})$ [1] has been proposed to allow different stakeholders to deploy local 5G networks. This helps in achieving high capacities [3] context specific services and content in these environments, to complement the MNO's offerings. The uOs need to provide flexibility, privacy, and customization in the network to serve the local needs of its customers which can include one or multiple tenants, where a tenant is a group of $\mathrm{uO}$ customers that have a unique requirement of tailored services. The 5G local networks are deployed in such a way that a $\mathrm{uO}$ could serve its own restricted customer set and/or could act as a host [4] for customers of MNOs by providing them services in a specific location.
For enabling the emergence of a large number of local 5G uOs into the mobile communication market, it is necessary to define the contractual relationships between MNOs, uOs and tenants, for different deployments. Since uO networks share several similarities with national roaming networks [5] and networks with mobile virtual network operators (MVNOs) [6], it is natural to refer to these networks for proposing the features of contractual relationships in uO networks. In [7], different operating models and features of contracts between MNOs and MVNOs, and the factors affecting their relationship, were discussed. The work in [5], described roaming agreements and the technological constraints that affect those agreements. A significant aspect of designing contracts in these networks is to define the optimal prices when establishing contracts. In case of national roaming networks too, the amount that MNO customers need to pay their home operators for accessing the roaming services is referred to as the retail price, and the prices which the MNOs of the visitor network need to be paid by the MNO of the home network, is called as the wholesale price [8]. In [9], different methods were proposed to determine optimal prices and profits in roaming networks. In the same way, in a $\mathrm{uO}$ network where the customers of an MNO are served by the $\mathrm{uO}$, it is necessary to evaluate the optimal prices for accessing the services of the $\mathrm{uO}$.

Another significant factor that influences the contractual relationships in MVNO and roaming networks, is competition. In [10], the authors showed that competition in roaming prices leads to the creation of a mutually beneficial relationship between the MNO and the MVNO. It is therefore, essential to analyze the impact of different levels of competition in $\mathrm{uO}$ networks and their role in determination of optimal prices. While designing features of these contractual relationships, it is also necessary to take into consideration, the new properties of $5 \mathrm{G}$ networks and beyond, such as multi-tenancy [11], network slicing [12] and dynamic service-level agreement (SLAs) [13]. These aspects of uOs' service agreements with the MNOs and tenants, keeping the new features of $5 \mathrm{G}$ and beyond into account, have not been explored before and is therefore important to study.

The main contributions of this paper are summarized below.

- Firstly, we define different contractual relationships for different deployment models in local 5G uOs networks, 


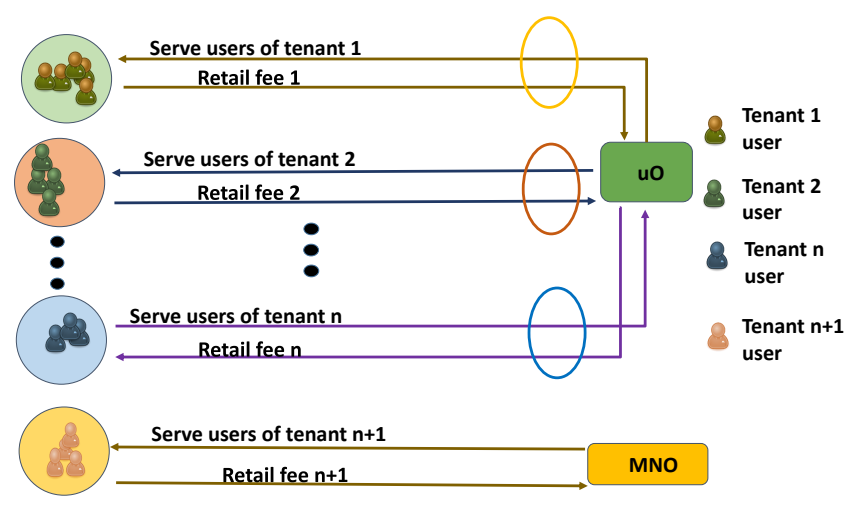

Fig. 1. Closed network relationships of uO, and different tenants, in presence of MNO exclusively serving tenants.

taking into account the properties of future $5 \mathrm{G}$ networks.

- We define competition in 5G uO networks and propose optimal wholesale pricing mechanisms depending on whether there is cooperation or no cooperation among the $\mathrm{uO}$ and the $\mathrm{MNO}$ in determination of this price.

- Finally, considering different scenarios of traffic flow from MNOs to uOs for an open network, the equilibrium wholesale prices are evaluated analytically and using numerical computations, to determine the effect of competition and share of MNO traffic served by the uO, on the equilibrium wholesale price.

The rest of the paper is structured as follows. Firstly, we describe the $\mathrm{uO}$ deployments and the resulting relationships in Section II and discuss competition in micro operator networks in Section III. Then we present illustrative examples of competition and pricing mechanisms in micro operator networks in Section IV, and in Section V we draws conclusions and outline possible avenues for future work.

\section{Micro Operator Deployments And Resulting RELATIONSHIPS}

The local 5G networks deployed by uOs can have different configurations depending on the customers they serve, due to which the possibilities of contractual relationships between uOs, MNOs, and tenants can vary. Therefore, it is necessary to analyze each deployment and their corresponding properties in order to understand the relationships among the entities. There are three deployment configurations of uO networks: closed, open, and mixed networks [14]. It is important to clarify that we assume that the customers of the MNOs pay the retail fee to their home operators for accessing the services of the $\mathrm{uO}$ whereas the tenant users or the customers of the $\mathrm{uO}$ pay their retail fee to the $\mathrm{uO}$ directly for their services. The wholesale fee is only paid by the MNOs to the $\mathrm{uO}$ for serving the MNO customers. We describe the contractual relationships for these deployments in the following sub-sections.

\section{A. Closed Network}

A closed $\mathrm{uO}$ network is a private network where network resources are allocated by the $\mathrm{uO}$ to individual tenants within the

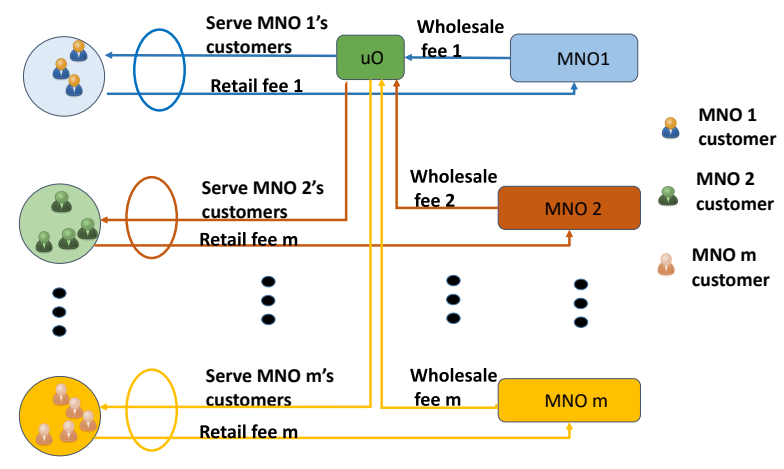

Fig. 2. Open network relationships between uOs, MNOs and customers of MNOs for agreements with unidirectional traffic flow.

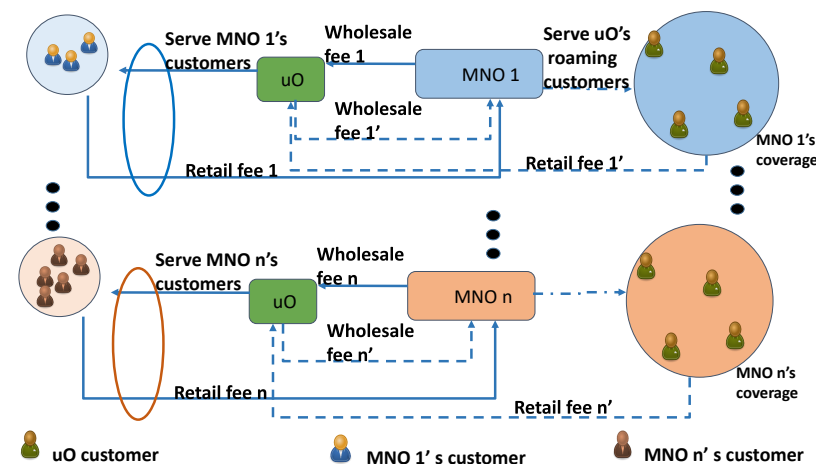

Fig. 3. Open network relationships between uOs, MNOs and customers of MNOs for agreements with reciprocal traffic flow.

network, according to the services they request for (as shown in Fig. 1). The uOs implement network slicing and serves every tenant with network slices. This way multiple tenants are served with diverse tailored services. Due to the different requirement of services for the tenants, different retail fee will be charged from every tenant. Therefore, contracts need to be defined between the uOs and tenants in this deployment. For example, a private network covering a hospital can serve different tenants, where the users belonging to a tenant have a unique set of requirements of services, and will therefore pay the $\mathrm{uO}$ a retail fee unique to the services provided by the $\mathrm{uO}$. This fee should reflect the degree of differentiated services, the quality and locations where their services are available, etc. In addition, it is also necessary to introduce authentication mechanisms in these networks, so that only users belonging to a tenant are able to access the network slice allocated by the $\mathrm{uO}$ for that tenant.

Alternatively, MNOs too are locally deployed and investments are made to develop infrastructures that offer tailored services for a tenant. Fig. 1 shows a closed network with the contractual relationships between the uOs, MNOs and the tenants.

\section{B. Open Network}

In an open uO network deployment, the uO serves $\mathrm{MNO}$ customers within a locality which requires that the $\mathrm{uO}$ forms contracts with the MNO. For example, the MNO can buy 


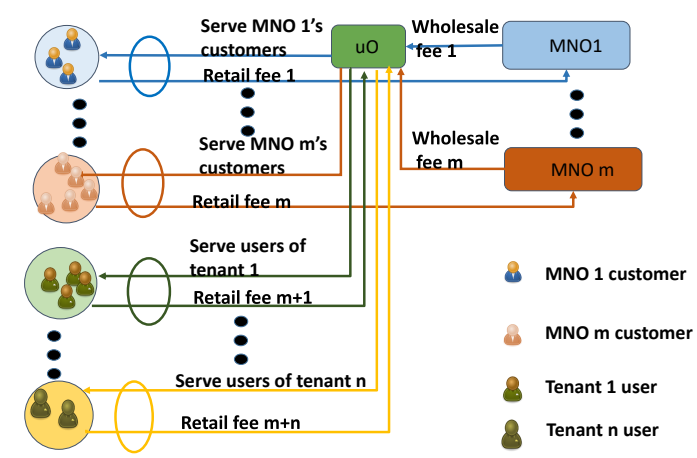

Fig. 4. Mixed network relationships between uO, MNOs, tenant users, and customers of MNOs.

the services from a $\mathrm{uO}$ instead of investing in building a network in that location. The uOs network can potentially serve the customers of a single MNO or customers of multiple MNOs. Since the customers of the MNOs are interested in the services offered by the uOs, it is necessary for MNOs to form contracts with the uOs. The uOs implements network slicing to provide network slices to different MNOs, which creates multiple contracts between the $\mathrm{uO}$ and the MNOs (as shown in Figs. 2 and 3). The MNOs' benefit from this relationship with uOs is good quality coverage services for its customers and savings in investments to be used for building its own network.

Due to different ways of negotiation in contracts between the uOs and the MNOs, two configurations of open network deployments can arise. They are (a) one way access of uO's network (Fig. 2) and (b) two way roaming access between MNO and uO's network (Fig. 3). In one way access of uO's network, for the customers of MNO that are served by the $\mathrm{uO}$, the MNO offers a wholesale payment to the $\mathrm{uO}$. In two way roaming access between $\mathrm{MNO}$ and $\mathrm{uO}$, the $\mathrm{uO}$ serves the customers of the MNO and its own customers has the opportunity to be served by the MNO when present within the MNO's far greater coverage area.

\section{Mixed Network}

In a mixed $\mathrm{uO}$ network deployment, the $\mathrm{uO}$ serves both MNO customers and tenants within a specific area, and therefore has combined properties of open and closed networks. In these networks, contracts need to be defined between uOs, MNOs and the tenants. The uO offers different network slices to tenants and MNOs according the services they request for. This deployment represents a complete and general model for networks deployed by uOs where the closed and open deployments are only the special cases of this model. These properties of mixed $\mathrm{uO}$ network deployments are shown in Fig. 4.

\section{COMPETITION IN Micro OPERATOR Networks}

Next, we discuss different possibilities of competition that can emerge in $\mathrm{uO}$ networks, which will potentially influence

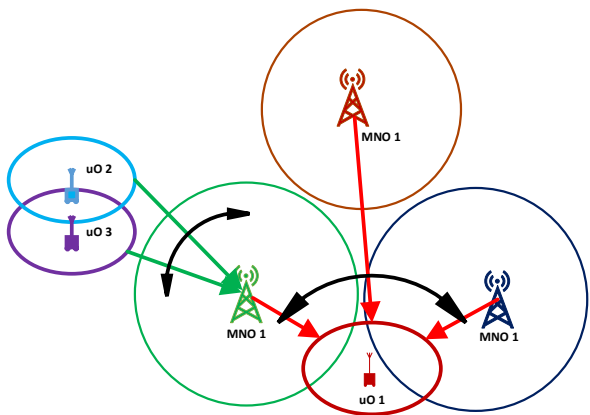

Fig. 5. Competition in micro operator networks.

the prices which the customers need to pay, and the level of innovation in services which the customers can have access to. In local $5 \mathrm{G} \mathrm{uO}$ networks, mainly two kinds of competition can occur, namely at the (a) MNO level and (b) at uO level. When a single $\mathrm{uO}$ can provide services to $\mathrm{MNO}$ customers in a specific area, several MNOs would like to avail that service by forming contracts with the concerned $\mathrm{uO}$. This can be referred to as competition at the MNO level. In Fig. 5, MNOs 1, 2 and 3 compete for the services of the uO 1. In [15], contractual relationships and pricing mechanisms based on competition between MNOs were described. On the other hand, when several uOs can offer services to the MNO customers in a common area, there is a possibility of competition among these $\mathrm{uOs}$ to form contracts with MNO resulting in attracting the MNO's traffic to one of the uO's network. Competition at the $\mathrm{uO}$ level is illustrated in Fig. 5, where uOs 2 and 3 compete for the services of the MNO 1. In addition to the $\mathrm{uO}-\mathrm{MNO}$ relationship, competition at the uO level can also impact the uO-tenant relationship. Multiple uOs in a location with different customized set of services can compete to serve the tenant users. We focus here, however, on the influence of competition among uOs, on the uO-MNO relationship.

\section{A. Considered Cases of Competition}

We focus on the scenario where the presence of multiple uOs creates competition and influences the uO-MNO relationship. The uO's incentive to attract the MNO's customers can be monetary profits, or the benefit of its customers having access to MNO's mobile network. The MNOs can have the choice of selecting the $\mathrm{uO}$ that provides the best quality service at the minimum wholesale price. For reciprocal relationships between uOs and MNOs, the MNOs would prefer to form contracts with the uOs that returns more roaming traffic to be served by the MNO.

\section{B. Non-cooperative/Cooperative Wholesale Pricing Models}

For contracts between uOs and MNOs, the wholesale price for accessing the services of an $\mathrm{uO}$ can be set up according to nature of agreements between the two parties, based on the direction of flow of traffic. In case of a unidirectional flow of traffic agreement, a non-cooperative method of pricing mechanism can be designed where each $\mathrm{uO}$ sets a unique, linear 
wholesale price so that both the $\mathrm{uO}$ and the $\mathrm{MNO}$ individually maximizes their profits [8], [5]. This method comprises of a two-stage game: (i) in the first stage each uO independently sets a wholesale tariff to maximize their individual profits, and (ii) in the second stage, MNOs charge a mark-up on the wholesale prices set by the uOs, in order to maximize their profits. But for reciprocal agreements between the MNO and the $\mathrm{uO}$, where both the parties need to collaborate for the determination of common interconnection terms in order to serve their own customers, the wholesale price can be set up cooperatively to maximize their joint profit [8].

Next, we provide an example of an open uO network and apply a method that was used in [8] for national roaming that shows the influence of competitive pressures on the equilibrium wholesale price.

\section{Illustrative EXAMPlE OF COMPETITION AND} Pricing MECHANISM IN Micro Operator NetworkS

Here, we present a few scenarios of an open uO network to show the impact of competition and share of MNO traffic served by a $\mathrm{uO}$, on the optimal wholesale price in these networks.

\section{A. System Model for an Open uO network}

We focus on a simple case where two uOs in a location have the requisite resources to serve the customers of two MNOs that have their distinct customer base in a region. The uOs charge wholesale prices in return for the services that they are capable of offering the MNO customers. In this paper, we consider that if competition exists among uOs, the criterion of competition is the wholesale price charged by the $\mathrm{uO}$, although there can be other criteria for competition such as the degree of differentiated services offered, and the area of coverage offered for the customers served by the uO. We use the non-cooperative method of determining the optimal wholesale price because the uO-MNO relationship in an open network involves a unidirectional flow of traffic only. The demand for uO's services faced by each MNO is not affected by the price charged by the competing MNO. For simplicity and without loss of generality, we assume linear demand functions, therefore the demand for uO's services faced by an MNO $d_{r}$, is given by

$$
d_{r}=A-\epsilon P_{r},
$$

where $A$ is the constant demand which is influenced by factors other than the price for accessing uO's services, $\epsilon$ represents the overall market's price demand sensitivity, $P_{r}$ is the price every MNO's customer pays for accessing uO's services, and $A, \epsilon>0$. Secondly, in order to ensure the existence of a market, we assume that

$$
A-\epsilon m>0,
$$

where $m$ is the constant marginal cost a $\mathrm{uO}$ faces for providing services to the customers of the MNOs.

We consider three example scenarios: (a) random distribution of MNO's traffic, (b) discounted distribution of MNO's

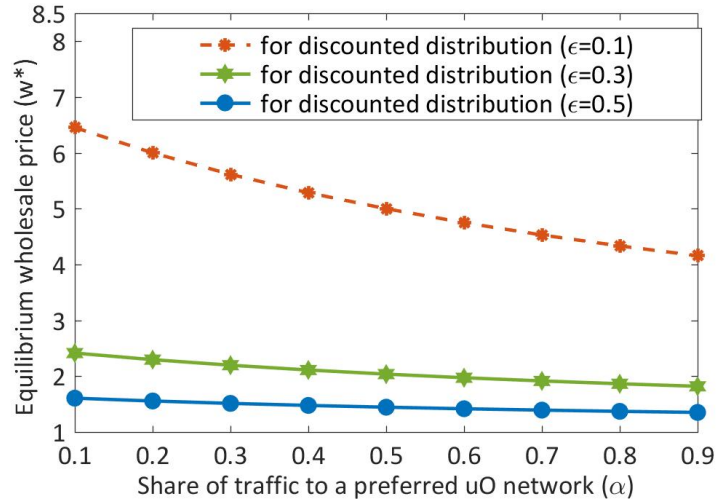

Fig. 6. Comparison of equilibrium wholesale prices for increasing $\alpha$.

traffic, and (c) competition. For the random distribution case, it is assumed that the customers of an MNO randomly choose either of the uOs for accessing $\mathrm{uO}$ services, resulting in a random distribution of traffic among the multiple uOs. For the discounted traffic scenario, we assume that a fraction of the traffic goes to a preferred $\mathrm{uO}$ and the remaining fraction of traffic is randomly distributed among the multiple uOs. The uO offers a discounted wholesale price based on the volume of traffic MNO steers towards it. The low discounted wholesale price applies only if the MNO directs its traffic to the $\mathrm{uO}$, orelse the high wholesale price is charged. For the competition case, we consider that each MNO steers the entire the roaming traffic to a preferred $\mathrm{uO}$ network, based on the result of competition among the uOs.

Next we perform a mathematical analysis of these scenarios to find the impact of competition and the share of MNO traffic served by a $\mathrm{uO}$, on the equilibrium wholesale price for uO's services.

\section{B. Mathematical Analysis and Results}

We evaluate the equilibrium wholesale price for the scenarios of random distribution, discounted distribution and competition, as described in Section IV-A. The three scenarios are described and optimal wholesale prices are found below, (a) Random distribution of MNO's traffic: When traffic steering is not used, the wholesale traffic from an $\mathrm{MNO}$ is usually randomly distributed between uOs. This represents a scenario where there is no competition at the wholesale level. We use the non-cooperative method of determining the optimal wholesale prices that was also used in roaming scenarios [5] and the wholesale price for a $\mathbf{u O} w_{n c}$ is set as

$$
w_{n c}=\frac{2 A+\epsilon m}{3 \epsilon} .
$$

(b) Discounted distribution of MNO's traffic: In agreements between MNOs and uOs, partial traffic steering can result in the $\mathrm{uO}$ offering a menu of wholesale prices $\left(w_{p c}^{L}, w_{p c}^{H}\right)$. The uO offers a discounted wholesale price based on the volume of traffic MNO steers towards it. The tariffs $w_{p c}^{L}$ and 


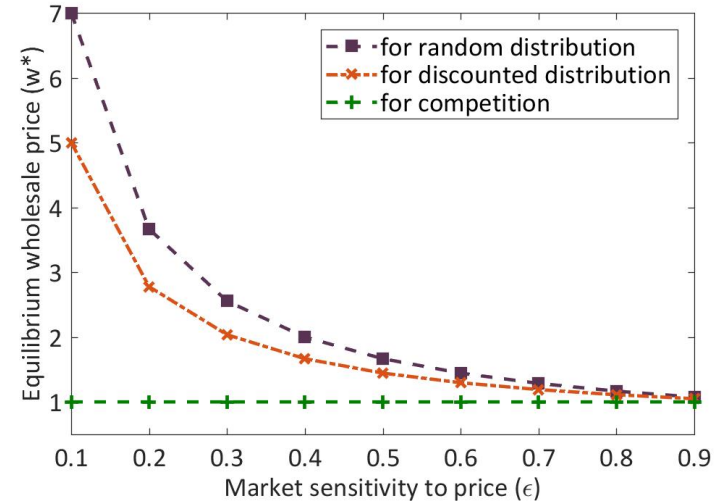

Fig. 7. Comparison of equilibrium wholesale prices with increasing $\epsilon$, for three different scenarios

$w_{p c}^{H}$ are such that $w_{p c}^{H}>w_{p c}^{L}$. The low discounted wholesale price applies only if the MNO directs its traffic to the $\mathrm{uO}$. Otherwise, the MNO is charged with $w_{p c}^{H}$. The equilibrium wholesale price $w_{p c}^{L}$ and $w_{p c}^{H}$ that MNOs are charged are evaluated by applying the method used in [8], and can be expressed as,

$$
w_{p c}^{L}=\frac{2 A+(3 \alpha+1) \epsilon m}{3 \epsilon(1+\alpha)}, w_{p c}^{H}=\frac{2 A+(3 \alpha-1) \epsilon m}{3 \epsilon(1-\alpha)} .
$$

where $\alpha \in(0,1)$ represents the share of MNO's traffic to a $\mathrm{uO}$ network, typically the one that charges the lowest $w_{p c}^{L}$. The remaining share of traffic $1-\alpha$, is randomly distributed between the two uOs. The scope of such contracts is to lower the wholesale prices of accessing uO's services.

(c) Competition among uOs: When MNO's traffic is completely steered towards the uOs, a competitive environment will emerge among the uOs to attract MNOs' traffic. As a result the MNOs can direct all its traffic completely to the $\mathrm{uO}$ that charges the least wholesale payment. Using the Bertrand model [16], this competition between the uOs will drive the wholesale price $w_{c}$ to the marginal cost $m$. Therefore,

$$
w_{c}=m \text {. }
$$

We perform numerical computations based on the evaluation of equilibrium wholesale prices for the three different scenarios using Equations (3), (4) and (5). Since a symmetrical model is considered, the equilibrium wholesale prices for both the uOs are evaluated to be equal. For conducting these computations, we have considered that the value of $A=1$, and value of $m$ is chosen such that it satisfies the relation $A-\epsilon m>0$. For our numerical computations, we take $m$ as equal to 2. In Fig. 6, by applying Equation (4), we show that with a higher fraction of traffic being steered to a preferred $\mathrm{uO}$ network, the equilibrium wholesale price decreases linearly. Moreover, higher the sensitiveness of a market to price, lower is the equilibrium wholesale price. In Fig. 7, we compare the equilibrium wholesale price to be paid to a $\mathrm{uO}$ for the three different scenarios as determined by Equations (3), (4) and (5). It is observed that the equilibrium price for random distribution of traffic among uOs always results in the highest wholesale price and competition leads to the lowest wholesale price among the three methods. Another important observation is that the different between the highest and the lowest equilibrium prices is maximum when the market sensitivity is low and this difference reduces with an increasing market sensitivity to price. Therefore, through this numerical analysis, we have shown that competition between uOs result in the least wholesale price for accessing uO's services.

\section{CONCLUSION}

In this paper, we have described new contractual relationships among different stakeholders and competitive environments in the mobile market due to emergence of local 5G networks deployed by entrant micro operators (uOs). uOs will provide services of wide variety to end users due to the introduction of novel features such as network slicing, dynamic SLAs, multi-tenancy etc.. This will result in more complicated relationships between the uOs, MNOs and tenants. We have focussed on these relationships and also have analyzed the different kinds of competition that will emerge in these networks.

Further, we have performed a mathematical analysis on an open $\mathrm{uO}$ network where competition between multiple uOs exists and studied the impact of competitive pressures on the equilibrium wholesale price that individually maximizes the profits of $\mathrm{MNO}$ and $\mathrm{uO}$. From our results, we have found that with competition, the uOs can attract the MNO customers and the equilibrium wholesale price can reduce to as low as the marginal cost. It is also found that lower the sensitiveness of a market demand to the price, higher is difference between the equilibrium wholesale prices for the scenario that of random distribution of traffic between uOs, and the scenario with competition between uOs. Moreover, higher the share of MNO traffic served by the $\mathrm{uO}$, lower is the equilibrium wholesale price for that $\mathrm{uO}$.

In the future, it is necessary to study the contractual relationships taking into account the features of $5 \mathrm{G}$ networks. One possible direction of work that will arise is the design of contracts where the properties of network slicing is applied in the $\mathrm{uO}$ networks. Also, pricing mechanisms for closed and mixed deployments of $\mathrm{uO}$ networks need to be studied and analyzed.

\section{REFERENCES}

[1] Marja Matinmikko and Matti Latva-aho and Petri Ahokangas and Veikko Seppanen, "On Regulations for 5G: Micro Licensing for Locally Operated Networks," Telecommunications Policy, vol. 42, no. 8, pp. 622635, September 2018.

[2] J. Zander, "Beyond the Ultra-Dense Barrier: Paradigm Shifts on the Road Beyond 1000x Wireless Capacity," IEEE Wireless Communications, vol. 24, no. 3, pp. 96-102, June 2017.

[3] D. H. Kang, K. W. Sung, and J. Zander, "High Capacity Indoor and Hotspot Wireless Systems in Shared Spectrum: a Techno-economic Analysis," IEEE Communications Magazine, vol. 51, no. 12, pp. 102109, December 2013.

[4] P. Ahokangas, S. Moqaddamerad, M. Matinmikko, A. Abouzeid, I. Atkova, J. F. Gomes, and M. Iivari, "Future Micro Operators Business Models in 5G," The Business \& Management Review, vol. 7, no. 5, p. 143, June 2016 
[5] R. Salsas and C. Koboldt, "Roaming Free?: Roaming Network Selection and Inter-operator Tariffs," Information Economics and Policy, vol. 16, no. 4, pp. 497-517, 2004

[6] S. Ulset, "Mobile Virtual Network Operators: a Strategic Transaction Cost Analysis of Preliminary Experiences," Telecommunication Policy, vol. 26, no. 9-10, pp. 537-549, 2002.

[7] C. Camarn and D. D. Miguel, "Mobile Virtual Network Operator (MVNO) Basics: What is Behind this Mobile Business Trend," Valoris viewpoint: Telecom Practice, October, 2008.

[8] P. Lupi and F. M. Manenti, "Traffic Management in Wholesale International Roaming: Towards a more Efficient Market?" Bulletin of Economic Research, vol. 61, no. 4, pp. 379-407, 2009.

[9] Organisation for Economic Cooperation and Development(OECD), "International Mobile Roaming Agreements," in OECD Digital Economy Papers, No. 223, June 2013.

[10] R. Dewenter and J. Haucap, “Access Pricing: An Introduction," Access Pricing: Theory and Practice, Amsterdam: Elsevier, 2007.

[11] K. Samdanis, X. Costa-Perez, and V. Sciancalepore, "From Network Sharing to Multi-tenancy: The 5G Network Slice Broker," IEEE Communications Magazine, vol. 54, no. 7, pp. 32-39, 2016.

[12] M. A. Habibi, B. Han, and H. D. Schotten, "Network Slicing in 5G Mobile Communication Architecture, Profit Modeling, and Challenges," 14th International Symposium on Wireless Communication Systems, Bologna, Italy, September 2017.

[13] L. Green, V. Mirchandani, I. Cergol, and D. Verchere, "Design of a Dynamic SLA Negotiation Protocol for Grids," in Proceedings of the first international conference on Networks for grid applications, 2007, p. 13.

[14] I. Badmus, M. Matinmikko-Blue, J. Walia, and T. Taleb, "Network Slice Instantiation for 5G Micro Operator Deployment Scenarios," European Confrence on Networks and Communications (EUCNC), Valencia, Spain, pp. 1-6, 2019.

[15] B. Barua, M. Matinmikko-Blue, and M. Latva-aho, "On Design of Contracts Between Traditional MNOs and Local 5G Micro Operators," International Telecommunications Society (ITS) European Conference, Helsinki, Finland, pp. 1-21, June, 2019.

[16] M. R. Baye and D. Kovenock, "Bertrand Competition," The New Palgrave Dictionary of Economics, pp. 1-7, 2017. 